\title{
Uso Profiláctico de Antibióticos en Cateterismo Umbilical en Recién Nacidos
}

\author{
Dr. Nathan Pulido S. ${ }^{1}$; Dra. Antonieta Montesinos $\mathrm{G}^{2}$; Srta. Marta Arriaza Z. ${ }^{3}$; \\ Dr. Patricio Esparza $A^{2}$ \\ Antibiotic prophylaxis after umbilical \\ vein catheterization
}

\begin{abstract}
Twenty nine term newborns were studjed at a Regional Hospital in Southern Chile to test the prophylactic value of antibiotics in infants undergoing blood exchange procedures by umbilical catheterism. Patients were asigned into two different groups, with or without antibiotic prophylaxis respectively. Complete biood counts and blood cultures were done in the third day after the procedure on both groups. There were 5 positive blood cultures among 15 newborns receiving antibiotics and 5 among 14 newborns managed without drugs. In retrospect and because no clinical nor hemathological evidence of infection was found in those patients, positive blood cultures were considered contaminated. It is concluded that in our experience the prophylactic use of antibiotics in term newborns undergoing umbilical catheterism is not useful in the prevention of secondary infections.

(Key words: Antibiotic prophylaxis. Secondary infections. Umbilical catheterism).
\end{abstract}

En Unidades de Recién Nacidos de Centros bien equipados no se usan rutinariamente antibióticos en pacientes sometidos a cateterismo de los vasos umbilicales $1 \cdot 2 \cdot 3-4 \cdot 5 \cdot 6$. Según las normas de atención del Recién Nacido del Ministerio de Salud, su empleo en estas situaciones

1. Becado de Pediatría, Universidad de la Frontera.

2. Neonatólogo, Unidad Recién Nacidos, Hospital Regional Temuco.

3. Interna Medicina, Unírersidad de Chile. depende del criterio del médico que realiza el procedimiento, $y$ las condiciones en que ésta se efectúe $^{7}$. En nuestro medio, habitualmente se indican antibióticos a los Recién Nacidos (RN) sometidos a procedimientos invasivos, como la cateterización de los vasos umbilicales, para disminuir el riesgo de una infección sistémica grave. Dicha conducta no tiene una justificación clara, ya que si bien es cierto que los RN son más susceptibles a las infecciones, no se ha demostrado que los antibióticos les otorguen protec- 
ciốn contra ellas en estos $\operatorname{casos}^{6-8-9}$.

El ptopósito de esta comunicación es evaluar la efectividad de los antibióticos en la prevención de las infecciones sistémicas del RN sometido a exanguineotransfusión o globuloforesis, mediante cateterismo venoso umbilical.

\section{MATERIAL Y METODO}

El estudio se realizó en la Únidad de Recién Nacidos del Hospital Regional de Temuco durante Julio y Agosto de 1984.

Fueron incluidos en él, todos los RN de término que, sin tener otra afección, debieron someterse a exanguineotransfusión o globuloforesis para tratar hiperbilirrubinemias o poliglobulinas, respectivamente. La indicación precisa y la técrica de los procedimientos son los habitualmente aceptados. Las exanguineotransfusiones fueron realizadas por hiperbilirrubinemia, cuyas causas fueron: incompatibilidad de grupo clásico: 10 ; causa no precísada: 9 ; poliglobulina: 3; incompatibilidad Rh: 1. Las globulofore sis se realizaron a RN con hematocrito igual o mayor de $70 \%$. Todos los procedimientos se realizaron por via venosa umbilical ${ }^{1} \cdot 2 \cdot 10$. 11 .

Los RN se distribuyeron alternativamente en 2 grupos, según orden de realización del procedimiento, uno de estudio, cuyos sujetos recibieron penicilina sódica $(50.000 \mathrm{U} / \mathrm{Kg} / \mathrm{d}$ ía) y gentamicina $(5 \mathrm{mg} / \mathrm{Kg} /$ dia) durante los tres días posteriores a la exanguineotransfusión o globuloforesis, y otro que no recibió antibióticos, sirviendo como control. Ambos recibieron los mismos cuidados habituales (fototerapia, alimentación, enfermeria, etc.).

Al tercer dia se tomaron hemogramas $y$ hemocultivos (dos) a todos los pacientes. Los he. mocultivos se tomaron por punción venosa periférica, siendo sembrados y procesados con técnicas habituales ${ }^{12}$. En ese momento, de acuerdo con la evidencia clínica y el hemograma se decidio si se suspendia, mantenía o iniciaba tratamiento con antibióticos. Si no existía evidencia de infección sistémica se concedió el alta el mismo día. Se eliminaron del estudio los R.N. en que fue necesario repetir el procedimiento (exanguineotransfusión o globuloforesisj. El resultado de los hemocultivos se leyó aproximadamente 7 días después de sembrada la muestra.

El diagnóstico de septicenia se estableció cuando, junto con el desarrollo de gérmenes en el hemocultivo, habia signos clínicos y hematológicos compatibles. Se consideraron signos clínicos sugerentes de septicemia; apneas y dificultad respiratoria $\sin$ causas evidentes, letargo, hipotermia, hepatoesplenomegalia, distensión abdominal, alteraciones del tránsito intestinal, púrpura, acidosis inexplicada, incremento ponderal deficiente, etc. ${ }^{1-2}-3-4-3$.

Se aceptó que el hemograma sugeria la posibilidad de infección sistémica, cuando en él se encontraban por lo menos 2 de los siguientes elementos: leucopenia (menos 5.000 por $\mathrm{mm}^{3}$ ), leucocitosis (mayor de 20.000 por $\mathrm{mm}^{3}$ ), indice de baciliformes neutrófilos mayor de 0.20 , trombocitopenia (menos de 100.000 por $\mathrm{mm}^{3}$ ), granulaciones toxicas o vacuolas en los neutrófi. $\operatorname{los}^{1-2}-3-4-5-13-14$.

El análisis estadístico para comparar la incidencia de síntomas sugerentes de septicemia y hemocultivos positivos entre ambos grupos se realizó mediante la Prueba de Diferencia de Proporciones (Z), considerando que no existía diferencia cuando el valor de $P$ era mayor que 0.05 .

\section{RESULTADOS}

Se estudiaron 29 RN de término, 16 de sexo femenino. Sus edades fluctuaron entre 1 y 10 días, con un promedio de 3,7 días en el momento del procedimiento, siendo de 3,2 días en el grupo que recibió antibióticos y 2,5 dias en el otro grupo. El peso, en el momento del procedimiento, varió entre 2.330 y 4.910 grs., promedio $3.180 \mathrm{grs}$. El peso promedio del grupo que recibió antibióticos fue 3.199 grs. y en los controles 3.169 grs. En el grupo de estudio. 8 pacientes eran de sexo femenino y 7 de sexo masculino. En el grupo control 8 eran de sexo femenino y 6 de sexo masculino.

Se practicaron 6 globuloforesis, 2 en el grupo de estudio y 4 en el de control y 23 exanguineotransfusiones, 13 y 10 respectivamente, que corresponden a 15 procedimientos en el grupo de estudio y 14 en el grupo control, encontrándose $5 \mathrm{RN}$ con hemocultivos positivos en cada uno: $33,3 \%$ y $35,7 \%$ respectivamente $(\mathrm{p}>0.45)$.

Ninguno de los RN tuvo manifestaciones clínicas o hematológicas que cumpliesen con los requisitos fijados por el estudio para ser considerados como evidencia de infección sistémica.

En ninguno de los RN del grupo control fue necesario indicar antibióticos posteriormente $y$ en todos los del estudio, fueron suspendidos al tercer dia.

Los gérmenes aislados fueron: Estafilococo Aureus 4, Estafilococo Epidermitis 4, Estreptococos sp 2, Pseudomona aeruginosa 1, y contaminado 1. Sólo I RN presentó los dos hemocultivos positivos, cada uno con gérmenes diferentes; en otro caso se aislaron dos gérmenes en un hemocultivo. 


\section{DISCUSION}

El cateterismo de los vasos umbilicales, especjalmente cuando se usa por períodos prolonga. dos se ha asociado a infecciones locales y sisté. micas 15, como son la contaminación del caté$\operatorname{ter}^{16}$, abscesos hepáticos ${ }^{17}$, endocarditis ${ }^{18}$ o septicemias, pero no se ha logrado demostrar que éstas se evitan con el uso profilàctico de antibióticos, sino con la realización del procedimiento con técnicas de asepsia adecuadas ${ }^{6-7}$.

En nuestro medio, dado que las condiciones de trabajo son diferentes a los de los paises desarrollados, se ha mantenido la práctica de indicar antibióticos a los RN sometidos a exan. guineotransfusión o globuloforesis por via umbi. lical, con el fin de disminuir el riesgo de infecciones sistemicas, aunque esta conducta no ha sido evaluada a nivel local.

En ninguno de los 2 grupos estudiados se encontró evidencia clínica o hematológica de septicemia, por lo que habría que considerar que los hemocultivos positivos pueden ser atribujdos a contaminación durante la extracción y manipulación de las muestras en el Laboratorio o a bacteremias asintomáticas. Cualquiera sea la explicación, los antibióticos no prođujeron diferencias significativas en la incidencia de hemocultivos positivos.

En todo caso, de tratarse de contaminación, habría que revisar las técnicas de enfermería y laboratorio, pues la proporción de hemocultivos positivos es excesiva. Los gérmenes aislados pueden formar parte de la flora cutánea de un RN hospitalizado. Llama la atención haber encontrado Estafilococo Aureus sólo en los RN que recibieron antibióticos, lo cual podría ser explicado por el efecto selectivo de éstos sobre los gérmenes resistentes.

No hubo diferencia en la incidencia de infecciones sistémicas entre el grupo que recibió antibiótico y el grupo control, por lo que se puede concluir que, en nuestro medio y en las condiciones habituales de trabajo, los antibióticos no parecen útiles en la prevención de septicemias en los RN de término, sin otras afecciones, que se someten a exanguineotransfusión o globuloforesis mediante cateterismo venoso umbilical.

\section{RESUMEN}

Para estimar la utilidad de los antibióticos en la prevención de septicemias en RN sometidos a exanguineotransfusiôn o globuloforesis mediante cateterismo umbilical, se estudiaron $29 \mathrm{RN}$ de término, en el Hospital Regional de Temuco, distribuidos al azar en dos grupos, uno que recibió antibióticos en la forma habitual y otro no. Se practicaron hemogramas $y$ hemocultivos al tercer día del procedimiento en ambos grupos. Se encontraron 5 hemocultivos positivos entre $15 \mathrm{RN}$ con antibióticos y 5 entre $14 \mathrm{sin}$ antibióticos. Todos los hemocultivos se consideraron contaminados porque no se acompañaron de signos clínicos ni hematológicos sugerentes de septicemia. Se concluye que en nuestro medio de trabajo no está indicado el uso profiláctico de antibióticos en los RN de término, sin otra morbilidad, que se someten a cateterismo umbilical.

\section{REFERENCIAS}

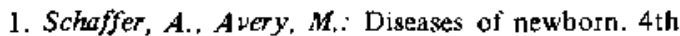
edition. Philadelphia, USA. W.B. Saunders Company. 1977 .

2. Klaus and Fonarof: Care of high-risk neonate. Second edition. Philadelphia, USA. W.B. Saunders Company, 1979.

3. Rizzardini, M.: Neonatología, Vol. I. Santiago, Chile. Editorial Andrés Bello. 1980.

4. Nelson, Voughan, McKay: Textbook of Pediatrics. 11 th edition. Philadelphia, USA. W.B. Saunders Company. 1979.

5. Rudolph, A.: Pediatrics. 7th edition. Norwalk, USA. Appleton. Century. Crofts. 1982.

6. Harris, M., Polin, R.: Neonatal septicemia. Pediatr. Clin. North. AM. 30: 251, 1983.

7. Texto guia y normas de atención del R.N. Ministetio de Salud Pública. Santiago, Chile. 1981.

8. Van Vket, O.. Gupta, $d .:$ Prophilactic antibiotics in umbilical artery catheterization in newborn. Arch. Dis. Child. 48: 296, 1973.

9. Perinatal infections. Ciba Foundation. Simposium 77, 1981. Pág. 239.

10. Kitferman, J., Phibbs, R., Tooley, W.: Catheterization of umbilical ressels in newborn infant. Pediatr. Clin. North. AM. 17: 895, 1970.

11. Levin. Morris Moore: A practical guide to Pediatric intensive care. St. Louis, USA. The C.B. Mosby Company. 1979.

12. Procedimientos y técnicas de Laboratorio. Vol. I: Microbiología general. Ministerio de Sajud Prública de Chile. Santiago, Chîle. Editorial Universitaria. 1983.

13. Zipursky, A., Jaber, $H_{\text {.: }}$ The Hematology in bacterial infections in newborn infants. Clinic. Hematology. 7: 175, 1978 .

14. Oski, F., Naiman, L.: Hematologic problems in the newborn. Third edition. Philadelphia, USA. W.B. Saunders Company. Pág. 235. 1982.

15. Balagras, R., Bell, E., Edwards, L., Levin, S.: Risk of local and sistemic infections associated with umbilical vein catheterization; a prospective study in 86 newborn patients. Pediatrics 48: 359, 1971.

16. Krauss, A., Alber, R., Kannan, M.: Contamination of unbilical catheters in newborn. J. Pediatt. 77: 965.1970.

17. Brans, Y. Ceballos, R., Cassady, G.: Umbilical catheters and hepatic abces. Pediatrics 53: 264. 1974.

18. Symchych, P. Kraus, A., Winchester, P.: Endocarditis following intracardiac placement of umbilical venous catheter in neonates. J. Pediatr. 90: 287,1977 . 